\title{
Selective activation and functional significance of p38 $\alpha$ mitogen-activated protein kinase in lipopolysaccharide-stimulated neutrophils
}

\author{
Jerry A. Nick, ${ }^{1,5}$ Natalie J. Avdi, ${ }^{1}$ Scott K. Young, ${ }^{1}$ Lisa A. Lehman, ${ }^{1}$ Patrick P. McDonald, ${ }^{2}$ \\ S. Courtney Frasch, ${ }^{2}$ Marcella A. Billstrom, ${ }^{1,5}$ Peter M Henson, ${ }^{2,5}$ Gary L. Johnson, $, 3,4$ \\ and G. Scott Worthen ${ }^{1,4,5}$ \\ ${ }^{1}$ Department of Medicine, \\ ${ }^{2}$ Department of Pediatrics, \\ ${ }^{3}$ Division of Basic Sciences, and \\ ${ }^{4}$ Program in Molecular Signal Transduction, National Jewish Medical and Research Center, Denver, Colorado 80206, USA \\ ${ }^{5}$ Department of Medicine, and \\ ${ }^{6}$ Department of Pharmacology, University of Colorado School of Medicine, Denver, Colorado 80262, USA
}

Address correspondence to: Jerry A. Nick, National Jewish Medical and Research Center, 1400 Jackson Street, Denver, Colorado 80206 , USA. Phone: (303) 398-1420; Fax: (303) 398-1851; E-mail: nickj@njc.org

Received for publication September 17, 1998, and accepted in revised form February 1, 1999.

\begin{abstract}
Activation of leukocytes by proinflammatory stimuli selectively initiates intracellular signal transduction via sequential phosphorylation of kinases. Lipopolysaccharide (LPS) stimulation of human neutrophils is known to result in activation of $\mathrm{p} 38$ mitogen-activated protein kinase (MAPk); however, the upstream activator(s) of $\mathrm{p} 38 \mathrm{MAPk}$ is unknown, and consequences of p38 MAPk activation remain largely undefined. We investigated the MAPk kinase (MKK) that activates p38 MAPk in response to LPS, the p38 MAPk isoforms that are activated as part of this pathway, and the functional responses affected by $\mathrm{p} 38 \mathrm{MAPk}$ activation. Although MKK3, MKK4, and MKK6 all activated p38 MAPk in experimental models, only MKK3 was found to activate recombinant p38 MAPk in LPS-treated neutrophils. Of p38 MAPk isoforms studied, only $\mathrm{p} 38 \alpha$ and $\mathrm{p} 38 \delta$ were detected in neutrophils. LPS stimulation selectively activated $\mathrm{p} 38 \alpha$. Specific inhibitors of $\mathrm{p} 38 \alpha$ MAPk blocked LPS-induced adhesion, nuclear factorkappa B (NF- $\mathrm{kB}$ ) activation, and synthesis of tumor necrosis factor- $\alpha$ (TNF- $\alpha$ ). Inhibition of $\mathrm{p} 38 \alpha \mathrm{MAPk}$ resulted in a transient decrease in TNF- $\alpha$ mRNA accumulation but persistent loss of TNF- $\alpha$ synthesis. These findings support a pathway by which LPS stimulation of neutrophils results in activation of MKK3, which in turn activates $\mathrm{p} 38 \alpha \mathrm{MAPk}$, ultimately regulating adhesion, NF- $\mathrm{KB}$ activation, enhanced gene expression of TNF- $\alpha$, and regulation of TNF- $\alpha$ synthesis.
\end{abstract}

J. Clin. Invest. 103:851-858 (1999).

\section{Introduction}

Stimulation of human neutrophils by lipopolysaccharide (LPS) elicits functional responses that are central to the pathogenesis of a number of human diseases. However, the intracellular signaling pathways used by neutrophils in response to proinflammatory stimuli have only begun to be elucidated. The recent delineation of the mitogen-activated protein kinase $(\mathrm{MAPk})_{1}$ superfamily provides a framework within which the response of neutrophils to LPS can be understood. MAPks are highly conserved signaling kinases that act to regulate cell growth, differentiation, and stress responses (1). At least three distinct families of MAPks exist in mammalian cells: the p42/44 extracellular signal-regulated kinase (ERK) MAPks, c-Jun $\mathrm{NH}_{2}$-terminal kinases (JNKs), and p38 MAPk (2-4). Our group and others (5, 6) have reported that p38 MAPk is activated in the neutrophil after LPS binding to CD14. In contrast, neither p42/44 (ERK) MAPks nor JNKs are activated by LPS stimulation of neutrophils under these conditions (5-7)

Activation of a MAPk is the final step in a three-part intracellular signal transduction cascade in which a
MAP/ERK kinase kinase (MEKK) or Raf activates (through phosphorylation) a MAP/ERK kinase (MEK or MKK), which in turn phosphorylates a specific tyrosine and threonine residue on a MAPk (1). At least three members of the MKK superfamily are capable of activating p38 MAPk. When overexpressed in cell lines, MKK3 (also termed MEK3), MKK4 (JNKK1), and MKK6 (MEK6) can all phosphorylate and activate p38 MAPk $(8,9)$. Four distinct isoforms of p38 MAPk have been identified in mammalian cells. The originally described human homolog of the Saccharomyces cerevisiae HOG1 kinase and the mouse p38 MAPk (2) is now referred to as p38 $\alpha$. Subsequently described isoforms include $\mathrm{p} 38 \beta$ with $74 \%$ amino acid identity to $\mathrm{p} 38 \alpha, \mathrm{p} 38 \gamma(60 \%$ identity to $\mathrm{p} 38 \alpha)$, and $\mathrm{p} 38 \delta$ $(57 \%$ identity to $\mathrm{p} 38 \alpha)(10,11)$. All of these isoforms share a common TGY motif in kinase subdomain VIII, where phosphorylation of a specific threonine and tyrosine residues is required for activation.

Once activated, the p38 MAPks appear capable of further signal transduction through phosphorylation of kinases, as well as by modulating functional responses through phosphorylation of transcription factors. 
MAPk-associated protein kinase-2 (MAPKAP-K2) and MAPKAP-K3 are activated directly by $38 \alpha \mathrm{MAPk}$, and they in turn can phosphorylate heat shock protein 27 (HSP27) $(3,6,12)$. Transcription factors directly phosphorylated by $\mathrm{p} 38 \alpha \mathrm{MAPk}$ include activated transcription factor-2 (ATF-2), serum response factor accessory protein-1, and myocyte enhancer factor $2 \mathrm{C}(13,14)$.

Most of our understanding of signal transduction in eukaryotic cells has risen from elegant transfection studies in cell lines. However, significant differences exist between the activation of signaling pathways in the neutrophil when compared with monocytes or cell lines $(13,15)$. As short-lived, terminally differentiated primary cells, neutrophils use rapid responses independent of transcriptional or translational mechanisms, as well as a limited repertoire of synthetic functions. Rapid responses to LPS include actin assembly and adherence. As a single stimulus, LPS is ineffective in evoking chemokinesis, chemotaxis, or the release of superoxide anion or granular enzymes. Functional responses to LPS that depend on de novo protein synthesis primarily consist of the release of cytokines (16).

We hypothesize that neutrophils use the p38 MAPk cascade to link proinflammatory stimuli to an array of functional responses. Additional specificity could occur through selective activation of MKK-family members and p38 MAPk isoforms. We studied the immediate upstream signaling events leading to activation of p38 MAPk, the relative activation of four p38 MAPk isoforms, and the functional consequences of p38 MAPk activation after stimulation with LPS. We report here that stimulation of neutrophils with LPS via CD14 initiates a signal that results in the activation of MKK3, which in turn phosphorylates and activates p38 $\alpha$ MAPk. Stimulation with LPS failed to activate $\mathrm{p} 38 \delta \mathrm{MAPk}$, and $\mathrm{p} 38 \beta$ and $\mathrm{p} 38 \gamma$ were not detected. After activation, p38 $\alpha$ MAPk regulates at least three distinctly different functions: adhesion, activation of nuclear factor-kappa $\mathrm{B}(\mathrm{NF}-\mathrm{\kappa B})$, and the synthesis of TNF- $\alpha$. Together, these results advance our understanding of an intracellular signaling pathway used by the human neutrophil in response to LPS.

\section{Methods}

Materials. Endotoxin-free reagents and plastics were used in all experiments. Neutrophils were isolated by the plasma Percoll method (17) and resuspended in Krebs-Ringer phosphate buffer with $0.2 \%$ dextrose at $\mathrm{pH} 7.2$, or in RPMI- 1640 culture medium (BioWhittaker, Walkersville, Maryland, USA). All experiments were done in the presence of $1 \%$ human heat-inactivated platelet-poor plasma. Aprotinin, leupeptin, Tris-HCL, Triton X-100, Igepal, PMSF, EDTA, EGTA, NP-40, and protein A-Sepharose were purchased from Sigma Immunochemicals (St. Louis, Missouri, USA), and $\left[\gamma^{-32} \mathrm{P}\right] A T P$ was purchased from Amersham Life Sciences Inc. (Arlington Heights, Illinois, USA). SK\&F86002 and SB203580 were provided by SmithKline Beecham Pharmaceuticals (King of Prussia, Pennsylvania, USA). ATF- $2_{1-110}$ and recombinant human p38 MAPk (rhp38 MAPk) were prepared as described previously $(5,17)$.

Neutrophil functional assays. Neutrophil adherence and actin assembly after stimulation with LPS were measured as described previously (17). Release of TNF- $\alpha$ was quantified by immunoassay (R\&D Systems, Minneapolis, Minnesota, USA). In vivo inhibition of $\mathrm{p} 38 \mathrm{MAPk}$ was performed by incubation of neutrophils in a range of concentrations of SK\&F86002 or SB203580 for $60 \mathrm{~min}$ at $37^{\circ} \mathrm{C}$. Statistical analysis of the significance of concentration-dependent inhibition for each functional response was performed by group mean values compared by one-way ANOVA. Preparation of nuclear extracts and the electrophoretic mobility shift assay (EMSA) to determine activation of NF- $\kappa B$ was performed as described previously (18).

P38 MAPk isoform antibodies. Specific antibodies against $\mathrm{p} 38 \alpha$, $\mathrm{p} 38 \beta, \mathrm{p} 38 \gamma$, and $\mathrm{p} 38 \delta$ and recombinant proteins for each were a kind gift of $\mathrm{H}$. Lichenstein (Amgen Inc., Boulder, Colorado, USA). Polyclonal antisera to the following peptides were prepared

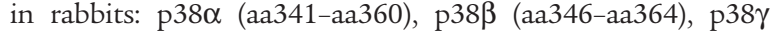
(aa321-aa339), and p38 (aa262-aa280). Peptides were conjugated to keyhole limpet hemocyanin and injected with CFA. Rabbit polyclonal antisera were also generated to full-length recombinant GST-flag-p38 $\delta$. For immunoprecipitation studies, IgG was purified from antiserum using the ImmunoPure IgG (Protein A) Purification Kit (Pierce Chemical Co., Rockford, Illinois, USA).

Immune depletion and immunoprecipitation assays. Neutrophils were stimulated and lysed (20 mM Tris-HCL [pH 7.5], 1\% Triton $\mathrm{X}-100,0.5 \%$ Igepal, $150 \mathrm{mM} \mathrm{NaCl}, 20 \mathrm{mM} \mathrm{NaF}, 0.2 \mathrm{mM}$ sodium orthovanadate, $1 \mathrm{mM}$ EDTA, $1 \mathrm{mM}$ EGTA). P38 $\delta$ was depleted from whole-cell lysates $(500 \mu \mathrm{l})$ containing $100 \mu \mathrm{g}$ cellular protein combined with $5 \mu \mathrm{g}$ purified anti-full-length $\mathrm{p} 38 \delta$ rotated for 6 or $12 \mathrm{~h}$ at $3^{\circ} \mathrm{C}$. Immune complexes were bound by the addition of $30 \mu \mathrm{l}$ of protein A-Sepharose and rotated for an additional $2 \mathrm{~h}$ at $3^{\circ} \mathrm{C}$. Bound Sepharose was removed by centrifugation $(14,000 \mathrm{~g}$ for $15 \mathrm{~s})$. The cleared supernatants were submitted to Western blot analysis. The $\mathrm{p} 38 \alpha$ and $\mathrm{p} 38 \delta$ bound to Sepharose were washed twice with lysis buffer and once in PBS and then submitted to Western blot analysis. Blots were probed for $\mathrm{p} 38 \alpha$ using an anti-p38 $\alpha$ (C20) antibody (Santa Cruz Biotechnology Inc., Santa Cruz, California, USA) and for p38 $\delta$ using anti-p38 $\delta$ (peptide) antisera. Tyrosine phosphorylation of p38 $\alpha$ and p38 was determined by immunoblotting with an anti-phosphotyrosine antibody (clone 4G10; Upstate Biotechnology Inc., Lake Placid, New York). Kinase activity of p38 $\alpha$ and p38 $\delta$ MAPks was assayed from immunoprecipitated samples by the ability to phosphorylate ATF-2 $2_{1-110}$ as described previously (17).

$M K K$ assays. Neutrophils were lysed with extract buffer (5) and combined with anti-MKK3 or anti-MKK4 antibodies (Santa Cruz Biotechnology Inc.) for immunoprecipitation studies. Bound Sepharose was resuspended in $25 \mu \mathrm{l}$ of kinase mix (17) with and without rhp38 MAPk. Reactions were terminated with $2 \times$ Laemmli buffer, and proteins were resolved with $10 \%$ SDS-PAGE and analyzed by phosphor screen autoradiography, with quantification of activity by ImageQuant (Storm Optical Scanner; Molecular Dynamics, Sunnyvale, California, USA) and qualitative analysis of kinase presence and phosphorylation by immunoblotting. Antibodies used to probe for MKK6 included MEK-6 (N-19) (K-19) (V-19) (Santa Cruz Biotechnology Inc.), anti-phospho MKK3/MKK6 (New England Biolabs Inc., Beverly, Massachusetts), and anti-MKK6 (full length), a kind gift of Amgen Inc. (Boulder, Colorado, USA).

Quantification of TNF- $\alpha m R N A$ by $R$ Nase protection assay. RNA was isolated from $30 \times 10^{6}$ neutrophils with Trizol Reagent (GIBCO BRL, Gaithersburg, Maryland, USA), as recommended by the manufacturer, and further purified by precipitation with $\mathrm{LiCl}$. The RNase protection assay (RPA) was carried out using a RiboQuant multiprobe kit (PharMingen, San Diego, California, USA). The RNase-protected duplexes were resolved on denaturing polyacrylamide gels and quantified by phosphorimaging and autoradiography.

\section{Results}

Coupled activation of MKK3 and $p 38$ MAPk in LPS-stimulated neutrophils. Because MKK3, MKK4, and MKK6 are 
$a$

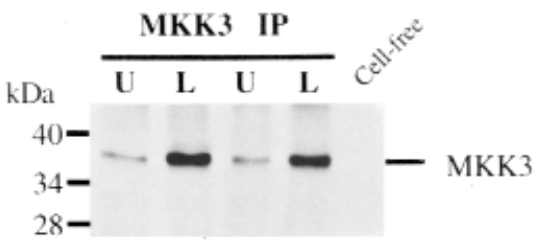

$\boldsymbol{b}$

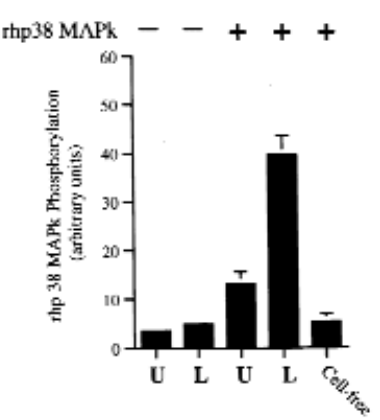

c

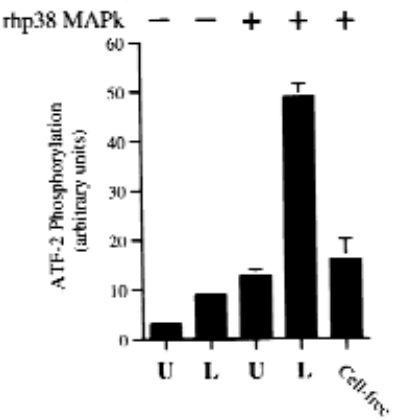

Figure 1

Coupled assay of MKK3 activation. Neutrophils were stimulated with LPS (100 ng/ml) for $20 \mathrm{~min}$ at $37^{\circ} \mathrm{C}$. (a) Phosphorylation of MKK3. MKK3 was immunoprecipitated from cell lysates of LPS-stimulated $(L)$ or unstimulated $(U)$ neutrophils. Lysates were submitted to SDS-PAGE and Western blotting with an anti-phosphorylated MKK3 antibody. A cell-free mixture that did not contain immunoprecipitated MKK3 is shown to control for intrinsic activity of the rhp38 MAPk. Blot is representative of three experiments. (b) Activation of MKK3. ${ }^{2} \mathrm{P}$ phosphorylation of rhp38 MAPk from blots was quantified by phosphor screen autoradiography. Lysates in the absence of rhp38 MAPk (lanes 1 and 2) were compared with lysates in the presence of rhp38 MAPk (lanes 3 and 4) and a cell-free control to demonstrate specific activation of MKK3 in response to LPS as determined by ${ }^{32} \mathrm{P}$ phosphorylation of rhp38 MAPk. (c) Coupled activation of rhp38 MAPk by activated MKK3. The ability of activated MKK3 to activate rhp38 MAPk was determined by the ability of phosphorylated rhp38 MAPk to phosphorylate the substrate ATF- $2_{1-110 .}{ }^{32} \mathrm{P}$ phosphorylation of ATF-2 $1-110$ from blots was quantified by phosphor screen autoradiography. Lysates in the absence of rhp38 MAPk (lanes 1 and 2) represent baseline phos-

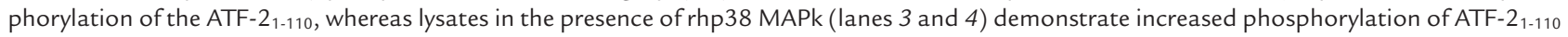
via coupled activation of rhp38 MAPk by activated MKK3 in response to LPS. The cell-free control quantifies intrinsic activity of the rhp38 MAPk and is equivalent to the unstimulated lysate in lane 3. Plots depict mean values and SEM from three consecutive experiments expressed in arbitrary units. ATF, activated transcription factor; LPS, lipopolysaccharide; MAPk, mitogen-activated protein kinase; MKK, MAPk kinase.

each capable of activating p38 MAPk, we first determined which of these MKKs activates p38 MAPk in the LPS-stimulated neutrophil. Stimulated neutrophils were lysed, and MKK3 was recovered by immunoprecipitation. Activation of MKK3 was determined by its phosphorylation and its ability to phosphorylate and activate rhp38 MAPk. Simultaneous assessment of the activation of the rhp38 MAPk was determined through its ability to phosphorylate ATF-2. The immunoprecipitated MKK3 was incubated with rhp38 MAPk in the presence of ATF-2 and [ $\left.{ }^{32} \mathrm{P}\right] \mathrm{ATP}$, followed by separation with sodium dodecyl sulfate-polyacry- lamide gel electrophoresis (SDS-PAGE). Neutrophils stimulated with LPS demonstrated phosphorylation of MKK3 (Fig. 1a) that corresponded to activation of MKK3, as assessed by phosphorylation of rhp38 MAPk (Fig. 1b). Activation of p38 MAPk requires specific phosphorylation of the $\mathrm{Tyr}^{182}$ residue. Thus, to prove phosphorylation of rhp38 MAPk by MKK3 was specific, assessment of activation of rhp38 MAPk was required. Phosphorylation of rhp38 MAPk by MKK3 resulted in its activation, as demonstrated by the ability of the phosphorylated rhp38 MAPk to in turn phosphorylate ATF-2 (Fig. 1c). Restaining of the a

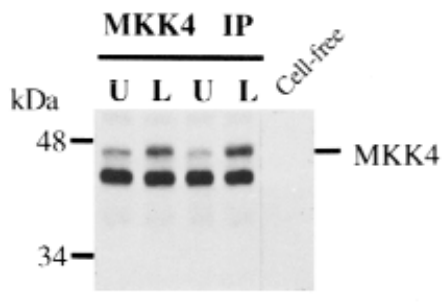

$\boldsymbol{b}$

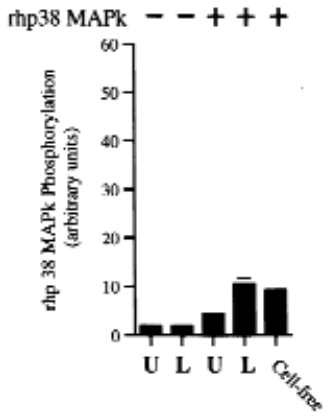

$\boldsymbol{c}$

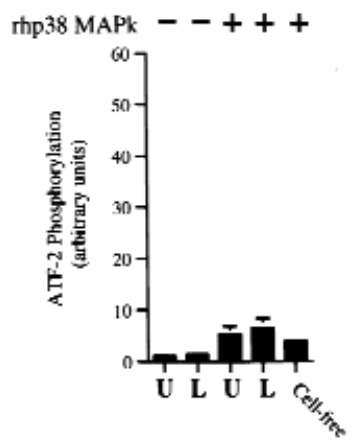

Figure 2

Coupled assay of MKK4 activation. Neutrophils were stimulated with LPS (100 ng/ml) for $20 \mathrm{~min}$ at $37^{\circ} \mathrm{C}$. (a) Phosphorylation of MKK4. Under identical conditions to the experiments shown in Fig. 1, MKK4 was immunoprecipitated from cell lysates and submitted to SDS-PAGE and Western blotting with an anti-phosphorylated MKK4 antibody. Blot is representative of three experiments. (b) Activation of MKK4. ${ }^{32} \mathrm{P}$ phosphorylation of rhp38 MAPk from blots was quantified by phosphor screen autoradiography. Lysates in the absence of rhp38 MAPk (lanes 1 and 2) were compared with lysates in the presence of rhp38 MAPk (lanes 3 and 4) and a cell-free control to demonstrate the lack of specific activation of MKK4 in response to LPS as determined by ${ }^{32} \mathrm{P}$ phosphorylation of rhp38 MAPk. (c) Coupled activation of rhp38 MAPk by MKK4. The ability of MKK 4 to activate rhp38 MAPk was determined by the ability of phosphorylated rhp38 MAPk to phosphorylate the substrate ATF-21-110. Lysates in the absence of rhp38 MAPk (lanes 1 and 2) represent baseline phosphorylation of the ATF-2 $1_{1-11}$, whereas lysates in the presence of rhp38 MAPk (lanes 3 and 4 ) demonstrate little increase in phosphorylation of ATF-2 $1-110$ via coupled activation of rhp38 MAPk by MKK4 in response to LPS. The cell-free control quantifies intrinsic activity of the rhp38 MAPk and is equivalent to the unstimulated and LPS-stimulated lysates in lanes 3 and 4. Plots depict mean values and SEM from three consecutive experiments expressed in arbitrary units. 
immunoblot with an anti-MKK3 antibody confirmed that equal amounts of MKK3 were immunoprecipitated under each condition (not shown).

MKK4 and MKK6 do not mediate p38 MAPk activation in LPS-stimulated neutrophils. Under identical conditions, MKK4 was immunoprecipitated from untreated and stimulated neutrophils. A detectable increase in phosphorylation of MKK4 was observed in neutrophils exposed to LPS when compared with unstimulated cells (Fig. 2a). However, no significant phosphorylation of p38 MAPk by MKK4 occurred, as determined by ${ }^{32} \mathrm{P}$ incorporation into rhp38 MAPk (Fig. 2b). In turn, no significant increase in rhp38 MAPk activity was seen as determined by ${ }^{32} \mathrm{P}$ phosphorylation of ATF-2 (Fig. 2c).

MKK6 is also capable of robust p38 MAPk activation; however, MKK6 (unlike MKK3 and MKK4) appears to have a high degree of cell-type specificity $(9,19)$. It has been reported (19) that in human leukocytes, MKK6 gene expression is undetectable, despite the fact that the cDNA encoding MKK6 was originally isolated from human T cells. Western blots performed on neutrophil whole-cell lysates using a panel of MKK6-specific antibodies (see Methods) failed to detect the protein when compared with myocyte and monocyte lysates (data not shown), suggesting that MKK6 is not constitutively expressed in human neutrophils.

LPS stimulation of neutrophils results in activation of $p 38 \alpha$, but not $p 38 \delta$, MAPk. Western blots of neutrophil lysates were probed with antibodies against p38, $\mathrm{p} 38 \gamma$, and p38 . Immunoblotting with antisera raised against a 19base peptide of $\mathrm{p} 38 \delta$ demonstrated a single band of the appropriate molecular weight. Identification of this band as p38 $\delta$ was supported by selective immunodepletion analysis of the neutrophil lysate (Fig. $3 a$ ). In contrast, immunoblotting with antibodies against $\mathrm{p} 38 \beta$ and $\mathrm{p} 38 \gamma$ failed to reveal the presence of these isoforms (data not

Figure 3

LPS stimulation selectively activates p38 $\alpha$ MAPk, but not p38 $\delta$ MAPk. (a) Identification of $\mathrm{p} 38 \delta \mathrm{MAPk}$ in neutrophil whole-cell lysates (WCL) by immunodepletion. Neutrophil lysate were subjected to SDS-PAGE and Western blotting with anti-peptide p38 $\delta$ antisera (lane 1). Confirmation of the identity of the p $38 \delta$ MAPk band was achieved by immunoprecipitating $\mathrm{p} 38 \delta$ from the lysate using a purified anti-fulllength $\mathrm{p} 38 \delta$ antibody. A significant decrease in the proposed $\mathrm{p} 38 \delta$ band is observed after $6 \mathrm{~h}$ (lane 2 ) and $12 \mathrm{~h}$ (lane 3) of immunodepletion. (b) Immunoprecipitation of p38 $\alpha$ and p38 MAPk. Neutrophils stimulated with LPS $(100 \mathrm{ng} / \mathrm{ml})$ for $25 \mathrm{~min}$ at $37^{\circ} \mathrm{C}(\mathrm{L}), \mathrm{H}_{2} \mathrm{O}_{2}$ $(1 \mathrm{mM})$ for $20 \mathrm{~min}$ at $37^{\circ} \mathrm{C}(H)$, or unstimulated $(U)$ were lysed, and p38 $\alpha$ and p38 MAPk were immunoprecipitated and separated by SDS-PAGE. Western blots were probed with antisera specific for $\mathrm{p} 38 \alpha$ and $\mathrm{p} 38 \delta$ MAPk to demonstrate equivalent amounts of immunoprecipitation for each condition studied. (c) Tyrosine phosphorylation of p38 $\alpha$ and p38 $\delta$ MAPk. Blots from $b$ were reprobed with an anti-phosphotyrosine antibody capable of reacting with phosphorylated tyrosine residues from both $\mathrm{p} 38 \alpha$ and $\mathrm{p} 38 \delta$ MAPk. (d) Activation of $\mathrm{p} 38 \alpha$ and p38 MAPk. Neutrophils subjected to identical conditions as in $b$ and $c$ were lysed, and p38 $\alpha$ and p $38 \delta$ MAPk was immunoprecipitated and combined with ATF- $2_{1-110}$ in the presence of [32P]ATP. The peptide was subjected to SDS-PAGE, and the degree of ${ }^{32} \mathrm{P}$ phosphorylation of ATF-2 ${ }_{1-110}$ was assessed by autoradiography of the blot. Each panel is representative of three consecutive experiments. shown). Failure to detect $\mathrm{p} 38 \beta$ and $\mathrm{p} 38 \gamma$ supports earlier reports of tissue specificity, as expression of $\mathrm{p} 38 \beta$ and p38 $\gamma$ mRNA is negligible in peripheral leukocytes (10).

Activation and phosphorylation of $\mathrm{p} 38 \alpha$ and $\mathrm{p} 38 \delta$ were assessed simultaneously in lysates from neutrophils stimulated with LPS or $\mathrm{H}_{2} \mathrm{O}_{2}$. Both $\mathrm{p} 38 \alpha$ and $\mathrm{p} 38 \delta$ were immunoprecipitated from cell lysates after stimulation and were resolved by SDS-PAGE. A Western blot probed with antibodies against $\mathrm{p} 38 \alpha$ and $\mathrm{p} 38 \delta$ demonstrated equivalent amounts of kinase for each condition (Fig. 3b). The blots were then restained with an antibody capable of detecting tyrosine phosphorylation of both p $38 \alpha$ and p38 (Fig. 3c). As shown previously, stimulation with LPS or $\mathrm{H}_{2} \mathrm{O}_{2}$ resulted in significant phosphorylation of $\mathrm{p} 38 \alpha$ MAPk. In contrast, LPS failed to phosphorylate p38 MAPk. $\mathrm{H}_{2} \mathrm{O}_{2}$, a potent activator of $\mathrm{p} 38 \delta$ when transfected into cells lines $(10,11)$ was seen to be capable of inducing phosphorylation of $\mathrm{p} 38 \delta$ in the neutrophil. Activation of p38 $\alpha$ and p $38 \delta$ was determined by immunoprecipitating each isoform from stimulated and unstimulated cells and combining the kinases with ATF-2 (a suitable substrate for both) in the presence of [32P]ATP. Corresponding to the pattern of tyrosine phosphorylation, stimulation with LPS failed to activate $\mathrm{p} 38 \delta$, whereas $\mathrm{H}_{2} \mathrm{O}_{2}$ was shown to be a potent activator of the isoform (Fig. $3 d$ ). Together, these results suggest that under the conditions studied, $\mathrm{p} 38 \alpha$ alone is activated in response to LPS stimulation of the neutrophil.

Inbibition of 38 MAPk selectively modulates rapid neutrophil responses to LPS. The pyridinyl imidazoles, SK\&F86002 and SB203580, have been demonstrated to inhibit p38 MAPk activity with insignificant effect on other mammalian signaling kinases $(4,11-13,17)$. SB203580 is now recognized to inhibit only the $\alpha$ and $\beta$ isoforms of p38 MAPk (11). To identify functional

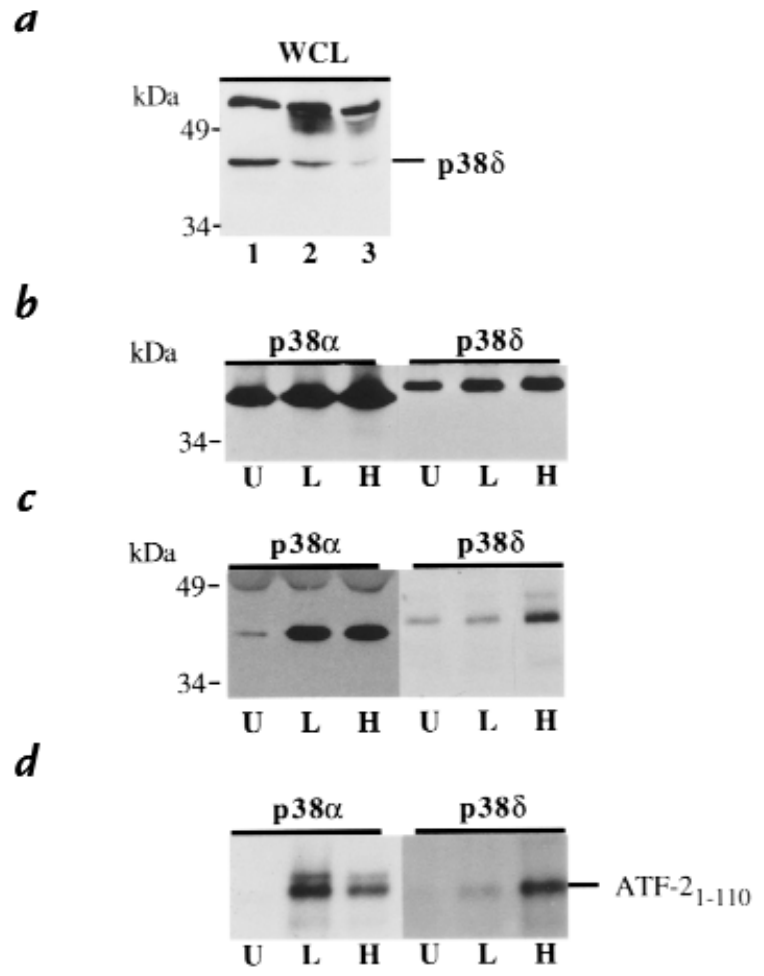




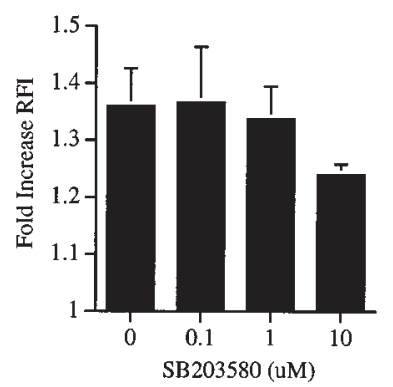

$\boldsymbol{b}$

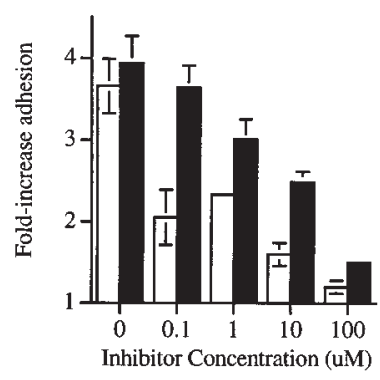

$\boldsymbol{C}$

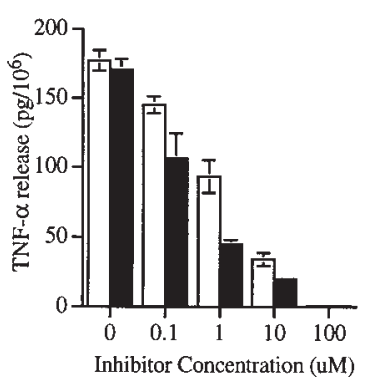

Figure 4

Effect of p $38 \alpha$ MAPk inhibition on LPS-induced rapid neutrophil responses. Neutrophils suspended in SK\&F86002 (open bars) or SB203580 (filled bars) over a range of concentrations for $60 \mathrm{~min}$ at $37^{\circ} \mathrm{C}$ were then stimulated with LPS $(100 \mathrm{ng} / \mathrm{ml})$ at $37^{\circ} \mathrm{C}$. (a) Effect of in vivo inhibition of p38 $\alpha$ MAPk on actin assembly. The -fold increase in relative fluorescence index (RFI) of LPS-stimulated neutrophils ( 5 min) compared with unstimulated cells was plotted for each concentration of SB203580. The plot represents mean activity and SEM for three independent experiments. The relationship between SB203580 concentration and inhibition of actin assembly was not statistically significant $(P=0.55)$. (b) Effect of in vivo inhibition of p $38 \alpha$ MAPk on neutrophil adhesion. The -fold increase in adhesion of LPS-stimulated neutrophils (30 min) compared with unstimulated cells was plotted for each concentration of inhibitor. The plot represents mean values and SEM for six experiments. The relationship between the concentrations of SK\&F86002 or SB203580 and inhibition of adhesion is significant $(P<0.0001)$. (c) Effect of p38 $\alpha$ MAPk inhibition on LPSinduced release of TNF- $\alpha$. The quantity of TNF- $\alpha$ released per $10^{6}$ neutrophils stimulated with LPS (120 min) was plotted for each concentration of inhibitor. The plot represents mean values and SEM of three consecutive experiments. The relationship between the concentrations of SK\&F86002 or SB203580 and inhibition of TNF- $\alpha$ release is significant $(P<0.0001)$. TNF, tumor necrosis factor.

responses to LPS that depend on p38 $\alpha$ MAPk activation, neutrophils were pretreated with SK\&F86002 or SB203580 followed by stimulation with LPS. The ability of neutrophils to assemble F-actin appears to be largely independent of p38 $\alpha$ MAPk activation, with no inhibitor effect noted except at the highest concentration studied (Fig. 4a). In contrast, pretreatment with the p38 MAPk inhibitors resulted in a significant, concentration-dependent decrease in neutrophil adhesion in response to LPS (Fig. 4b).

Inbibition of $p 38 \alpha$ MAPk activation downregulates TNF$\alpha$ gene expression and release in LPS-stimulated neutrophils. Inhibition of p38 MAPk by pyridinyl imidazoles has been shown to reduce TNF- $\alpha$ release in monocytes and certain cell lines (4). We therefore investigated whether $\mathrm{p} 38 \alpha \mathrm{MAPk}$ is a component of the signaling pathway leading to TNF- $\alpha$ release in neutrophils. Cells treated with a range of concentrations of either SK\&F86002 or SB203580 were then stimulated with LPS, and subsequent quantification of TNF- $\alpha$ release was performed by enzyme-linked immunosorbent assay. Both p38 MAPk inhibitors reduced in a dosedependent fashion the ability of LPS to elicit the secretion of TNF- $\alpha$ (Fig. $4 c$ ).

In view of the fact that the release of cytokines by activated neutrophils is preceded by enhanced de novo protein synthesis and gene expression (16), we next investigated the effect of MAPk inhibitors toward mRNA and TNF- $\alpha$ protein accumulation in LPS-stimulated cells. To determine whether inhibition of $\mathrm{p} 38 \alpha$ MAPk activation might modulate TNF- $\alpha$ mRNA steady-state levels in LPS-stimulated neutrophils, the cells were suspended in the presence or absence of SB203580 and stimulated with LPS for 30 and $60 \mathrm{~min}-$ utes. TNF- $\alpha$ mRNA was quantified by RPA. Stimulation with LPS elicited a significant increase in TNF- $\alpha$ mRNA, which could be detected as early as 30 minutes after LPS addition. Pretreatment of the cells with SB203580 caused a 50\%-60\% inhibition of the LPSinduced TNF- $\alpha$ mRNA accumulation at the 30-minute time point (Fig. 5, $a$ and $b$ ). However, this effect of SB203580 on TNF- $\alpha$ mRNA was transient: it was no longer observed by 60 minutes (Fig. 5, $a$ and $b$ ) or at later times (not shown). To determine whether inhibition of $\mathrm{p} 38 \alpha$ MAPk resulted in a posttranslational block of processing or release of TNF- $\alpha$, we quantified cell-associated TNF- $\alpha$ from LPS-stimulated cells after inhibition of $\mathrm{p} 38 \alpha \mathrm{MAPk}$. Pretreatment of neutrophils with SB20580 did not result in an intracellular or membrane-associated accumulation of TNF- $\alpha$ (data not shown). Together these results indicate that inhibition of $\mathrm{p} 38 \alpha$ MAPk downregulates TNF- $\alpha$ release by acting primarily at the level of (or before) translation.

Inhibition of $p 38 \alpha$ MAPk activation prevents NF- $\kappa B$ activation in LPS-stimulated neutrophils. Stimulation of neutrophils with LPS has been reported to result in NF$\mathrm{\kappa B}$ activation (18), and this transcription factor is known to regulate TNF- $\alpha$ gene expression in many cell types. We therefore investigated whether p38 $\alpha$ MAPk inhibition might modulate NF- $\mathrm{KB}$ activation in neutrophils. Cells were pretreated with SB203580 and subsequently stimulated with LPS before EMSA analysis of nuclear extracts. LPS-induced NF- $\mathrm{KB}$ activation was almost completely inhibited in neutrophils pretreated with the p38 MAPk inhibitor (Fig. 6a). The possibility that SB203580 might directly interfere with NF- $\mathrm{KB}$ binding to our NF- $\kappa \mathrm{B}$ probe was ruled out because incubation of nuclear extracts from LPStreated cells in the presence of SB203580 resulted in no difference in DNA-binding activity (Fig. 6b). Inhibition of LPS-elicited NF- $\kappa B$ by SB 203580 may contribute to the transient decrease in TNF- $\alpha$ mRNA accumulation observed in LPS-stimulated neutrophils after SB203580 pretreatment. 
a

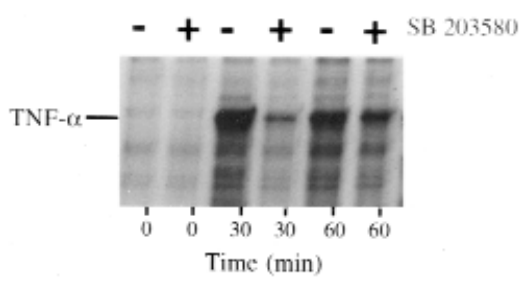

$\boldsymbol{b}$

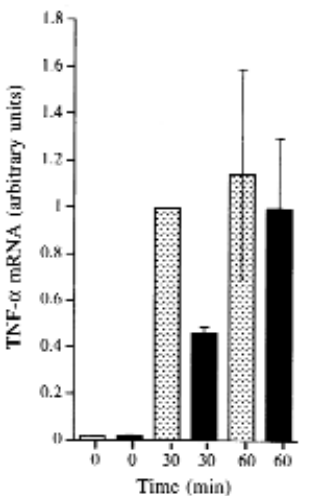

$\boldsymbol{C}$

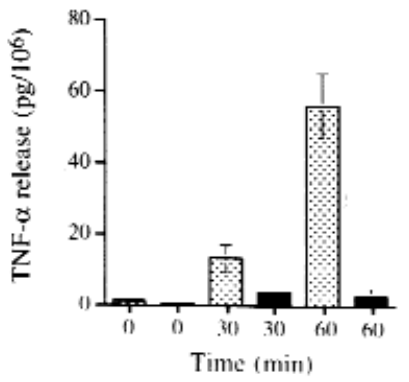

Figure 5

Quantification of TNF- $\alpha$ mRNA by RPA. (a) RPA autoradiograph of TNF- $\alpha$ mRNA at 0,30 , and 60 min after stimulation with LPS (100 ng/ml) at $37^{\circ} \mathrm{C}$ in the presence $(+)$ and absence $(-)$ of SB203580 $(10 \mu \mathrm{M})$. An increase in TNF- $\alpha$ mRNA seen after 30 min of stimulation (lane 3$)$ is substantially reduced in cells treated with SB203580 (lane 4). At 60 min, the quantity of TNF- $\alpha$ mRNA in the untreated and SB203580-treated cells (lanes 5 and 6 ) is equivalent. The blot is representative of three consecutive experiments. (b) Normalized plot of TNF- $\alpha$ mRNA synthesis as quantified by RPA. Amount of TNF- $\alpha$ mRNA present for each condition was expressed as a fraction of GAPDH present for each sample to correct for potential differences in sample loading. Each value was then normalize to the amount ofTNF- $\alpha$ mRNA present at 30 min of LPS stimulation in the untreated neutrophils (lane 3) to correct for variability in response between donors. After 30 min of LPS stimulation, SB203580-treated neutrophils (closed bar) demonstrated $42 \%$ of the TNF- $\alpha$ mRNA present in the untreated cells (hatched bar). After 60 min of stimulation, no significant difference is seen. The panel represents mean values and SEM of three consecutive experiments. (c) Release of TNF- $\alpha$ under conditions studied. TNF- $\alpha$ released from the neutrophils studied in $b$ was assayed. The quantity of TNF- $\alpha$ released per $10^{6}$ neutrophils was plotted for each time in the presence (closed bars) or absence (hatched bars) of SB203580. The panel represents mean values and SEM of the three consecutive experiments described for $b$. GAPDH, glyceraldehyde phosphate dehydrogenase; RPA, RNase protection assay.

\section{Discussion}

Stimulation of human neutrophils with physiological concentrations of LPS in the presence of LPS-binding protein (LBP) is initiated through binding of CD14. Our group and others $(5,20)$ have demonstrated that LPS-induced activation of p38 MAPk and subsequent release of TNF- $\alpha$ can be nearly eliminated by blocking the binding of LPS to CD14. After binding of the LPS-LBP complex to CD14, the signal must pass through the plasma membrane via a companion membrane-spanning protein, because CD14 does not have an intracellular component. Human homologs of the Toll receptor in Drosophila are transmembrane proteins that appear capable of such a function. When transfected into cell lines, toll-like receptor (TLR) 2 promotes LPS signal transduction, which is enhanced by coexpression of CD14 (21). In the LPS-resistant $\mathrm{C} 3 \mathrm{H} / \mathrm{HeJ}$ and $\mathrm{C} 57 \mathrm{BL} / 10 \mathrm{ScCr}$ strains of mice, inability to respond to LPS has been attributed to mutations in the gene expressing TLR4 (22). The role of TLRs in a

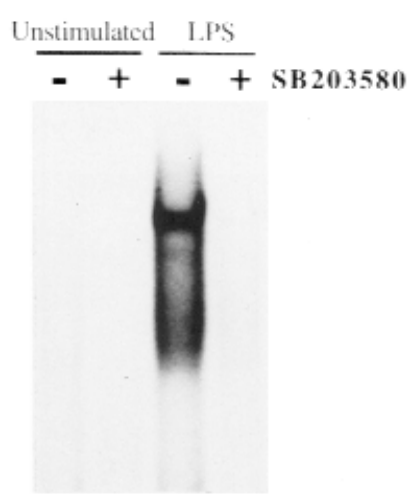

$\boldsymbol{b}$

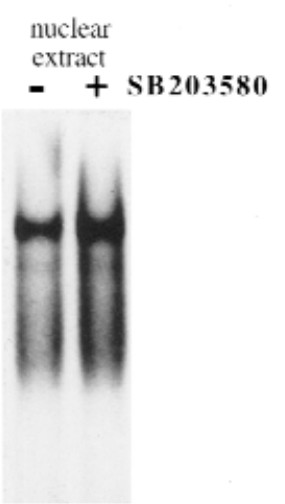

$\boldsymbol{c}$

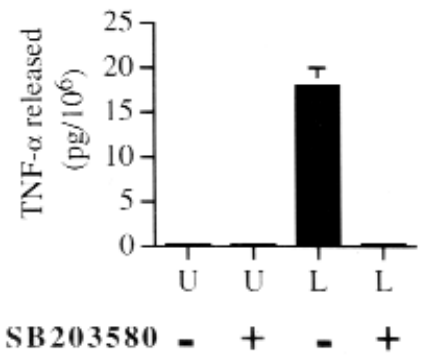

Figure 6

Inhibition of NF-KB activation by SB203580-induced inhibition of p38 $\alpha$ MAPk. (a) Effect of in vivo inhibition of p38 $\alpha$ MAPk on NF-KB activation. Neutrophils were suspended in the presence or absence of SB203580 (10 $\mu \mathrm{M})$ for $60 \mathrm{~min}$ at $37^{\circ} \mathrm{C}$ and subsequently stimulated with LPS (100 $\left.\mathrm{ng} / \mathrm{ml}\right)$ for 20 $\min$ at $37^{\circ} \mathrm{C}$ or left unstimulated. Nuclear extracts were then prepared and analyzed in EMSA using an NF- $\kappa B$ oligonucleotide probe. This blot is representative of three consecutive experiments. ( $\boldsymbol{b}$ ) Lack of a direct effect of SB203580 toward the binding of NF- $\mathrm{\kappa B}$ to its cognate sequence. Nuclear extracts from LPS-stimulated neutrophils were coincubated with SB203580 (100 $\mu \mathrm{M})$ for $30 \mathrm{~min}$ at room temperature in the binding mixture, before EMSA analysis. This blot is representative of three consecutive experiments. (c) Release of TNF- $\alpha$ at the time of NF-KB activation. The quantity of TNF- $\alpha$ released per $10^{6}$ neutrophils from the samples studied in $a$ was plotted for each condition. Bars depict the mean value and SEM of TNF- $\alpha$ release (per $10^{6}$ neutrophils) from three independent experiments. EMSA, electrophoretic mobility shift assay; NF- $\kappa B$, nuclear factor-kappa $B$. 
the LPS-signaling pathway of human neutrophil has yet to be determined.

Although early intracellular signaling events in this pathway remain undefined, they are independent of protein kinase $C$ and do not utilize Ras, Raf, MEKK-1, or MEK1/MEK2 (5). Instead, we now show that LPSinduced activation of p38 MAPk occurs via MKK3 (independent of MKK4 or MKK6). Only the p38 $\alpha$ and p38 $\mathrm{MAPk}$ isoforms were detected in the neutrophil, and only $\mathrm{p} 38 \alpha$ was found to be activated in response to LPS. The activation of $\mathrm{p} 38 \alpha \mathrm{MAPk}$ contributes to functional responses including adhesion, NF- $\kappa \mathrm{B}$ activation, and the synthesis of TNF- $\alpha$. The exact role of p38 $\alpha$ MAPk in the complex series of events that lead to neutrophil adhesion is not known, but because adhesion occurs independent of protein synthesis, this regulation is likely to involve the phosphorylation of different substrates than the ones affecting the release of TNF- $\alpha$. A diagram depicting our present understanding of LPS-induced intracellular signaling in the neutrophil is shown in Fig. 7.

Through use of specific p38 MAPk inhibitors such as SK\&F 86002 and SB203580, several functional consequences of $\mathrm{p} 38 \alpha \mathrm{MAPk}$ activation have been identified. These pyridinyl imidazoles have been shown in vitro to inhibit specifically the $\alpha$ and $\beta$ isoforms of p38 MAP, with negligible effect on other important protein kinases and phosphatases $(4,11,12,17)$. Likewise, in vivo studies in whole cells demonstrated inhibition of p38 MAPk, with no effect on activation of JNKs and p42 ERK MAPks $(4,12)$. At higher concentrations, inhibition of certain JNK isoforms may occur; however, these kinases are not activated by LPS in the neutrophil under our experimental conditions (13). In human neutrophils, inhibition of p38 MAPk results in decreased superoxide anion production, reduction in adhesion and chemotaxis in response to fMLP $(17,23)$, and obstruction of interleukin (IL)-8 release in response to LPS (23).

Our data suggest that $\mathrm{p} 38 \alpha$ MAPk activation may influence at least two regulatory steps in the synthesis of TNF- $\alpha$. Inhibition of LPS-induced NF- $\kappa B$ may contribute to the transient decrease in TNF- $\alpha$ mRNA accumulation observed in LPS-stimulated neutrophils after pretreatment with SB203580. Thus, inhibition of transcriptional events might represent one of the mechanisms whereby p38 $\alpha$ MAPk inhibitors block TNF- $\alpha$ release. However, it must be emphasized that the decrease in TNF- $\alpha$ mRNA levels resulting from p38 MAPk inhibition is only observed during the first hour of LPS stimulation. This suggests that the sustained inhibition of TNF- $\alpha$ synthesis resulting from $\mathrm{p} 38 \alpha$ MAPk blockade must involve additional mechanisms, and it raises the possibility of a posttranscriptional role for $\mathrm{p} 38 \alpha \mathrm{MAPk}$ in the context of TNF- $\alpha$ production.

Consistent with our data, a number of studies have provided evidence for transcriptional and translational effects of p38 MAPk. In cell lines, p38 MAPk inhibitors have been reported to downregulate the expression of several genes. In particular, LPS-induced c-Jun mRNA in RAW 264.7 cells (14), TNF- $\alpha$-induced IL-6 mRNA in L929 cells (24), and IL-1-induced prostaglandin endoperoxide synthase- $2 \mathrm{mRNA}$ in primary monocytes (25) are reduced by inhibition of p38 MAPk. It is noteworthy that the genes encoding IL-6, TNF- $\alpha$, and prostaglandin endoperoxide synthase- 2 all depend on NF-KB (albeit to varying degrees) for inducible expression (26) and that both p38 MAPk and ERK MAPk have been reported to downregulate TNF$\alpha$-induced IL- 6 gene expression by interfering with NF-KB-dependent transcription in L929 cells (27).

Inhibition of LPS-stimulated IL- 1 and TNF- $\alpha$ production by p38 MAPk inhibitors in THP- 1 cells occurs at the translational level (4). In these cells, SK\&F86002 had little effect on TNF- $\alpha$ mRNA levels but instead was found to inhibit TNF- $\alpha$ mRNA translation by inducing a shift of TNF- $\alpha$ mRNA from polysomes (actively translated) to free mRNA (translationally inactive) (28). One possible mechanism by which p38 MAPk could regulate translation could be through the activation of MAPk-interacting kinase 1, which phosphorylates eukaryotic translation initiation factor eIF-4E (29) and is known to play a key role in the regulation of translation in mammalian cells.

Significant differences appear to exist between monocytes/macrophages and neutrophils with respect to the role of p38 MAPk in TNF- $\alpha$ synthesis. Recent reports in LPS-stimulated RAW 264.7 cells have demonstrated a regulatory effect of p42/44 (ERK) MAPk on TNF- $\alpha$ tran-

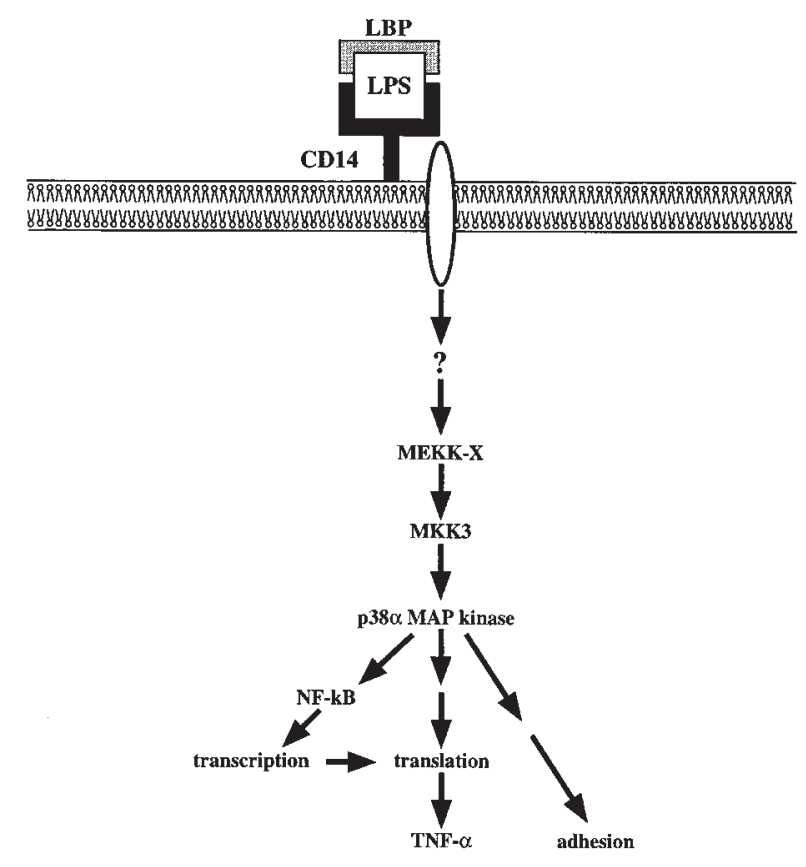

\section{Figure 7}

Scheme depicting proposed intracellular signaling pathways and functional consequences in response to stimulation of human neutrophils with LPS. Binding of CD14 by LPS in the presence of LBP initiates a signal that passes though a transmembrane spanning protein and a series of yet unknown upstream signaling events, leading to activation of the MKK3-p38 $\alpha$ MAPk cascade. The activator of MKK3 in this cell $(M E K K-X)$ is not known. Activation of $\mathrm{p} 38 \alpha \mathrm{MAPk}$ then results in rapid responses such as adhesion and activation of NF-KB. Synthesis of the TNF- $\alpha$ peptide is also dependent on $p 38 \alpha$ MAPk activation, in part through activation of NF- $\mathrm{KB}$, but primarily through regulation of translation. $L B S$, lipopolysaccharide-binding protein. 
scription, and a significant role of the MEKK1/MEK4/JNK cascade on TNF- $\alpha$ translation (30). In the mast cell line RBL-2H3, production of TNF- $\alpha$ is linked to activation of p42 MAPk, but not p38 MAPk (31). However, in human monocytes, a role for p38 MAPk activation in the induction of TNF- $\alpha$ and IL- $1 \beta$ release has been proposed $(4,32)$. In contrast, we and others have shown that the p42/44 (ERK) MAPk are not activated in human neutrophils in response to LPS $(5,7)$ and that the ability to activate JNKs diminishes as HL-60 promyelocytic cells differentiate into neutrophils (15). These differences support the observation that MAPk cascades may serve a wide range of purposes, depending on the cellular context and upstream regulation (1). A growing body of evidence supports the conclusion that regulatory influences of p38 MAPk can occur independent of protein synthesis or at the site(s) of transcription and/or translation to varying degrees in a gene-specific manner. Further specificity is conferred by the ability of different cell types to use the MAPk cascades in distinct manners in response to identical stimuli. These results underscore the importance of defining signaling pathways under physiological conditions in primary human cells.

\section{Acknowledgments}

We thank Carl Manthey for technical advice and encouragement. The work was supported by National Institutes of Health grants K08 HL-03657, HL-40784, HL-34303, HL-09640, and GM-30324. P.P. McDonald is a Centennial Fellow of the Medical Research Council of Canada.

1. Cobb, M.H., and Goldsmith, E.J. 1995. How MAP kinases are regulated. J. Biol. Chem. 270:14843-14846.

2. Han, J., Lee, J.-D., Bibbs, L., and Ulevitch, R.J. 1994. A MAP kinase targeted by endotoxin and hyperosmolarity in mammalian cells. Science. 265:808-811.

3. Rouse, J., et al. 1994. A novel kinase cascade triggered by chemical stress and heat shock which stimulates MAPKAP-2 and phosphorylation of the small heat shock proteins. Cell. 78:1027-1037.

4. Lee, J.C., et al. 1994. A protein kinase involved in the regulation of inflammatory cytokine biosynthesis. Nature. 372:739-746.

5. Nick, J.A., et al. 1996. Activation of a p38 MAPk in human neutrophils by LPS. J. Immunol. 156:4867-4875.

6. Nahas, N., et al. 1996. Tyrosine phosphorylation and activation of a new MAPk cascade in human neutrophil stimulated with various agonists. Biochem. J. 318:247-253.

7. Fouda, B.I., Molski, T.F.P., Ashour, M.S., and Sha'afi, R.I. 1995. Effect of LPS on MAPks and cytosolic phospholipase A2. Biochem. J. 308:815-822.

8. Dérijard, B., et al. 1995. Independent human MAP kinase signal transduction pathways defined by MEK and MKK isoforms. Science. 267:682-685.

9. Han, J., et al. 1996. Characterization of the structure and function of a novel MAPKK (MKK6). J. Biol. Chem. 271:2886-2891.
10. Wang, X.S., et al. 1997. Molecular cloning and characterization of a novel p38 MAPk. J. Biol. Chem. 272:23688-23674.

11. Goedert, M., et al. 1997. Activation of the novel stress-activated protein kinase SAPK4 by cytokines and cellular stresses is mediated by SKK3 (MKK6): comparison of its substrate specificity with that of other SAP kinases. $E M B O J .16: 3563-3571$.

12. Cuenda, A., et al. 1995. SB 203580 is a specific inhibitor of a MAPk homologue which is stimulated by cellular stresses and interleukin1. FEBS Lett. 364:229-233.

13. Whitmarsh, A.J., et al. 1997. Role of p38 and JNK MAPks in the activation of ternary complex factors. Mol. Cell. Biol. 17:2360-2372.

14. Han, J., Jiang, Y., Li, Z., Kravchenko, V.V., and Ulevtich, R.J. 1997. Activation of the transcription factor MEF2C by the MAPk p38 in inflammation. Nature. 386:296-299.

15. Frasch, S.C., et al. 1998. p38 MAPk-dependent and -independent intracellular signal transduction pathways leading to apoptosis in human neutrophils. J. Biol. Chem. 273:8389-8397.

16. Cassatella, M. 1995. The production of cytokines by polymorphonuclear neutrophils. Immunol. Today. 16-21.

17. Nick, J.A., et al. 1997. Common and distinct intracellular signalling pathways in human neutrophil utilized by platelet activating factor and FMLP. J. Clin. Invest. 99:975-986.

18. McDonald, P.P., Bald, A., and Cassatella, M.A. 1997. Activation of the NF-kB pathway by inflammatory stimuli in human neutrophils. Blood. 89:3421-3433.

19. Stein, B., Brady, H., Yang, M.X., Young, D.B., and Barbosa, M.S. 1996. Cloning and characterization of mek6, a novel member of the MKK cascade. J. Biol. Chem. 271:11427-11433.

20. Haziot, A., Tsuberi, B.-Z., and Goyert, S.M. 1993. Neutrophil CD14: biochemical properties and role in the secretion of TNF- $\alpha$ in response to LPS. J. Immunol. 150:5556-5565.

21. Yang, R.-B., et al. 1998. TLR-2 mediates LPS-induced cellular signalling. Nature. 395:284-288.

22. Poltorak, A., et al. 1998. Defective LPS signaling in $\mathrm{C} 3 \mathrm{H} / \mathrm{HeJ}$ and C57BL/10ScCr mice: mutations in Tlr4 gene. Science. 282:2085-2088.

23. Zu, Y.-L., et al. 1998. p38 MAPk Activation is required for human neutrophil function triggered by TNF- $\alpha$ or FMLP stimulation. $J$. Immunol. 160:1982-1989.

24. Beyaert, R., et al. 1996. The p38/RK MAPk pathway regulates IL-6 synthesis in response to TNF. EMBO J. 15:1914-1923.

25. Pouliot, M., et al. 1997. Inhibition of prostaglandin endoperoxide synthase- 2 expression in stimulated human monocytes by inhibitors of p38 MAPk. J. Immunol. 158:4930-4937.

26. Baeuerle, P.A., and Henkel, T. 1994. Function and activation of NFkappa B in the immune system. Annu. Rev. Immunol. 12:141-179.

27. Berghe, W.V., et al. 1998. p38 and ERK MAPk pathways are required for NF-kB p65 transactivation mediated by TNF. J. Biol. Chem. 273:3285-3290.

28. Prichett, W., Hand, A., Sheilds, J., and Dunningyon, D. 1995. Mechanism of action of bicyclic imidazoles defines a translational regulatory pathway for TNF- $\alpha$. J. Inflamm. 45:97-105.

29. Waskiewicz, A.J., Flynn, A., Proud, C.G., and Cooper, A.J. 1997. MAPks activate the serine/threonine kinases Mnk1 and Mnk2. EMBO J. 16:1909-1920.

30. Swantek, J.L., Cobb, M.H., and Geppert, T.D. 1997. JNK/SAPK is required for LPS stimulation of TNF- $\alpha$ translation: glucocorticoids inhibit TNF- $\alpha$ translation by blocking JNK/SAPK. Mol. Cell. Biol. 17:6274-6282.

31.Zhang, C., Baumgartner, R.A., Yamada, K., and Beaven, M.A. 1997. MAPk regulates production of TNF- $\alpha$ and release of arachidonic acid in mast cells. Indications of communication between p38 and p42 MAPks. J. Biol. Chem. 272:13397-13402.

32. Young, P., et al. 1993. Pyridinyl imidazoles inhibit IL-1 and TNF production at the protein level. Agents Actions. 39:C67-C69. 